\title{
Steady State and Multifrequency Phase Fluorometry Studies of Binding Site Flexibility in Related Antibodies ${ }^{\dagger}$
}

\author{
Gopi S. Mohan, Philip T. Chiu, ${ }^{\ddagger}$ Cathrine A. Southern, ${ }^{\S}$ and Patricia B. O’Hara* \\ Department of Chemistry, Amherst College, Amherst Massachusetts 01002
}

Received: February 13, 2004; In Final Form: May 26, 2004

\begin{abstract}
The fluorescence properties of related antibodies were characterized from proteins representing different time points in the murine antibody immune response (the period of time during which the selectivity and sensitivity to the small molecule hapten is enhanced several 100-1000-fold). Two of the antibodies studied are catalytic and bind covalently to a substrate analogue used to induce antibody production. The remaining three represent antibodies produced after primary, secondary, and tertiary immunizations with hapten. In the first series of experiments, steady-state fluorescence spectroscopy and multifrequency phase fluorometry were used to examine the fluorescence properties of the proteins' tryptophan residues. Though unique interpretations regarding the binding site differences are confounded by the intrinsic complexity of the system, analysis of the data reveals that while the lifetimes are similar for the different antibodies, the lifetime distributions seem to narrow as a function of antibody maturity. Hapten binding results in little change in the lifetimes but a marked narrowing of the lifetime distributions. In the second series of experiments, the steady state and multifrequency phase fluorometry data of Prodan [6-propionyl-2-(dimethylamino)naphthalene], a fluorescent hapten analogue, were analyzed for binding site differences. Excitation and emission spectra, lifetimes, and lifetime distributions were measured and demonstrated differences in the binding sites of antibodies collected at different stages of the immune response.
\end{abstract}

\section{Introduction}

Affinity maturation, an integral part of some organisms' response to infection, is the process by which the immune system produces increasingly specific and selective antibodies for a particular antigen after repeated exposures to that antigen. Antigens are large molecules that elicit an immune response, though only small regions of the antigen actually contact the binding site of an antibody. The structure of an antibody can be broken down into constant regions, which interact with other molecules involved in generating an immune response, and variable regions, which are responsible for binding antigen. A schematic representation of an antibody distinguishing the variable and constant regions is shown in Figure 1.

Specifically, affinity maturation involves the selection and proliferation of cells that produce antibodies with high affinity for a particular antigen. Upon subsequent exposures to the same antigen, antibody-producing "memory" cells stored in the bone marrow are "recalled" by the immune system. Some small fraction of the antibody-producing cells migrates to the spleen, where small modifications or "point mutations" are made to the genes for these recalled antibody-producing cells. Occasionally these mutations produce cell lines that express antibodies with higher binding affinity and selectivity. The mutated cells go through a selection process, where those producing antibodies with the highest affinity for antigen are allowed to proliferate while the cells producing weaker-binding antibodies die off.

\footnotetext{
† Part of the special issue "Richard Bersohn Memorial Issue".

* Corresponding author. E-mail: pbohara@amherst.edu. Telephone: 413542-2732. Fax: 413-542-2735.

$\doteqdot$ Current address: Department of Materials Science and Engineering, Northwestern University, Evanston, IL 60208.

$\S$ Current address: Department of Chemistry, College of the Holy Cross, Worcester, MA 01610.
}

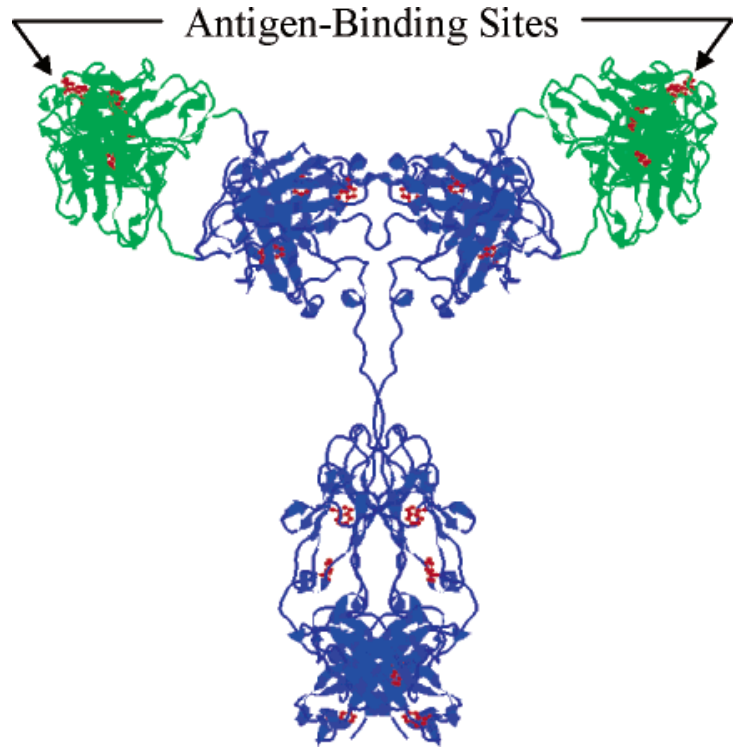

Figure 1. Schematic model of an intact antibody with the constant regions colored blue and the variable regions, which are specific for an antigen, colored green. The binding sites (two per molecule) are indicated with arrows at the very tips of the two arms of the antibody. Tryptophan residues are colored in red. The figure was drawn from a PDB file kindly provided by Eduardo A. Padlan, who combined the files $2 \mathrm{IG} 2 . \mathrm{PDB}^{35}$ and $1 \mathrm{FC} 2 . \mathrm{PDB}^{36}$ and modeled in the remainder of the structure. ${ }^{37}$

This entire process of recollection, modification, and selection is repeated after each antigen exposure, or "immunization," and results in successively more effective antibodies. ${ }^{1}$

It has been proposed that a key factor in increasing the binding affinity and selectivity of antibodies is structural annealing of 


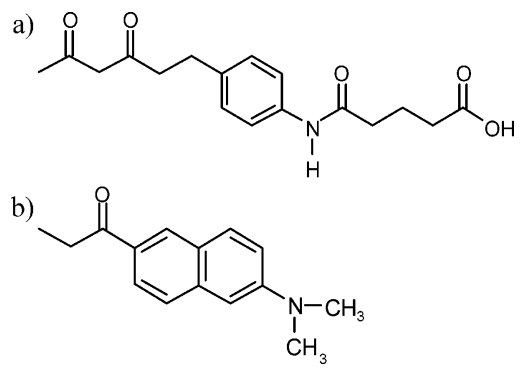

Figure 2. Chemical structures of (a) the diketone hapten used to elicit aldolase antibodies ${ }^{8}$ and (b) the fluorescent hapten analogue 6-propionyl-2-(dimethylamino)naphthalene (Prodan).

the binding site to specifically complement the antigen. ${ }^{2-5}$ This involves the introduction of mutations that reduce the flexibility of the binding site in such a way that it is preconformed to the molecular structure of the antigen. According to this hypothesis, binding sites in immature germ line antibodies can be characterized as being flexible with relatively low affinity $\left(K_{\mathrm{d}}\right.$ in the $\mu \mathrm{M}$ range) and a broad selectivity while mature antibodies are more rigid with exquisite selectivity and affinities 100- to 1000fold higher $\left(K_{\mathrm{d}}\right.$ in the $10-100 \mathrm{nM}$ range) than their germ line ancestors. The lack of flexibility in the more mature antibodies reduces the entropic cost to the antibody when the antigen binds, thereby leading to a higher binding affinity. Support for these ideas has come from thermodynamic studies of series of related antibodies using isothermal calorimetry ${ }^{6}$ and surface plasmon resonance spectroscopy. ${ }^{7}$ To further examine this hypothesis using fluorescence spectroscopy, we have examined a series of antibodies obtained from a library of antibodies collected at different stages of the immune response. These antibodies were created by immunization with the hapten, first designed by Wagner and co-workers, ${ }^{8}$ shown in Figure $2 \mathrm{a}$ (a hapten is a small organic molecule that binds in a specific fashion to an antibody and elicits an immune response when conjugated to a carrier protein).

The goal of this work is to see if the fluorescence properties of antibodies expressed at various stages of the immune response yield evidence for a decrease in the conformational heterogeneity of the antibody binding site as the maturity of the antibody increases. Toward that goal, fluorescence from both the native tryptophan (Trp) side groups and the fluorescent hapten analogue 6-propionyl-2-(dimethylamino)naphthalene (Prodan), ${ }^{9}$ shown in Figure $2 b$, were characterized as a function of binding.

Binding sites for all antibodies are rich in Trp residues, and the quenching of the steady-state fluorescence emission of these residues upon small molecule hapten binding is a standard method for the determination of relative binding affinities. ${ }^{10,11}$ Though this technique does yield relative binding affinities, it is plagued by low sensitivity due to the poor fluorescence quantum yield of Trp. Another confounding problem is the presence of background fluorescence from Trp residues in the constant regions of the antibody that do not show specific quenching upon hapten binding. These issues also complicate the examination of antibody binding site flexibility using Trp fluorescence lifetimes and lifetime distributions. Despite these important complications, the sensitivity of the Trp emission to binding makes it a natural choice as a potential reporter of the conformational heterogeneity of the binding site. We have used multifrequency phase fluorometry, a method of time-resolved fluorescence spectroscopy that allows fluorescence lifetimes to be determined, ${ }^{12}$ in an attempt to discern whether affinity maturation affects the Trp fluorescence lifetime or lifetime distribution. The distribution of lifetimes, or the range of lifetimes a fluorophore or a group of fluorophores displays, is a measure of the conformational heterogeneity of the system under study. ${ }^{13,14}$ These observations may show whether affinity maturation is accompanied by protein conformational selection as reported by the fluorescence of Trp at the binding site.

An alternative method for studying the heterogeneity of the system is to look at the conformational distribution of the hapten itself when bound to the different antibodies. Toward that end, we have examined the fluorescence properties of Prodan, a fluorescent hapten analogue. Results from this approach are not complicated by interfering signals from regions of the antibody distant from the binding sites. We examined the steady-state properties and the lifetimes and lifetime distributions of Prodan when bound to our panel of antibodies. The fluorescence properties of Prodan have been used previously to characterize hydrophobic binding sites in proteins. ${ }^{9,15-20}$ We have shown that Prodan binds specifically to the binding sites of the five monoclonal antibodies used in this study and not to our control antibody. Analysis of the fluorescence properties of Prodan can be used to characterize the changes in the binding site as a result of affinity maturation from the perspective of the bound ligand.

\section{Experimental Section}

A. Sample Preparation. Three of the monoclonal antibodies were produced by the following methodology. Antibodies were elicited in mice injected with the hapten shown in Figure 2a conjugated to a carrier protein by standard immunological methods. ${ }^{21}$ Animals were sacrificed either 12 days after the initial exposure to the hapten (primary antibody A3.1.1), 5 days after the first boost, (secondary antibody 2C26.1), or 5 days after the second boost (tertiary antibody 3.22), and antibodyproducing cells were isolated from the spleen. Tight-binding populations were selected, and standard hybridoma technology was used to create immortal cell lines, which could at any time be injected into the peritoneal cavity of a mouse to produce antibody-secreting tumors. Fluid from the animals containing a high concentration of antibody was purified using Pharmacia A/G purification columns. Protein purity was confirmed by gel electrophoresis. One of the commercially available monoclonal antibodies used here, 38C2 (Aldrich), was raised against the hapten shown in Figure $2 \mathrm{a} .{ }^{8}$ The other commercially available antibody, 84G3 (Aldrich), was raised against a structurally similar hapten. ${ }^{22,23}$ Both of these antibodies have catalytic aldolase functionality. ${ }^{8,22,23}$ A nonspecific antibody from mouse (murine IgG1, Kappa (MOPC 21), Sigma-Aldrich) was used to test for nonspecific interactions of the antibody with either the hapten or Prodan. Control tryptophan (Sigma) solutions were prepared to be $15 \mathrm{mg} / \mathrm{mL}$ to approximate the Trp concentration in our antibody studies. All solutions were made in phosphate buffered saline (PBS) of $0.010 \mathrm{M} \mathrm{Na}_{2} \mathrm{HPO}_{4}, 0.002 \mathrm{M} \mathrm{KH}_{2-}$ $\mathrm{PO}_{4}, 0.144 \mathrm{M} \mathrm{NaCl}$, and $0.003 \mathrm{M} \mathrm{KCl}$, adjusted to $\mathrm{pH}$ 7.2. In every case, this solution was filtered using a $0.2 \mu \mathrm{m}$ Acrodisc filter before use.

The hapten was synthesized according to published protocol. ${ }^{8}$ A stock solution of the hapten was made by dissolving $3.2 \mathrm{mg}$ of the hapten in $0.500 \mathrm{~mL}$ of acetonitrile (Optima grade, Fisher), and then diluting $1 / 400$ in filtered PBS to create a $50 \mu \mathrm{M}$ hapten stock solution that was then added to the antibody solutions. For 38C2, 84G3, and 3.22 (the tight-binding antibodies), 20 $\mu \mathrm{L}$ of the diluted stock was added per $150 \mu \mathrm{L}$ of antibody solution to create final concentrations of $5.9 \mu \mathrm{M}$ hapten and $2.9 \mu \mathrm{M}$ antibody. For the primary and secondary antibodies, the antibody concentration was the same, but the final concen- 
tration of hapten was higher to ensure saturation of the antibody binding sites. Complete binding occurred within $10 \mathrm{~min}$, at which point frequency domain measurements could be obtained.

Prodan [6-propionyl-2-(dimethylamino)naphthalene] (Molecular Probes) stock solutions were prepared by dissolving 4.55 $\mathrm{mg}$ of Prodan in $20.0 \mathrm{~mL}$ of methanol (Optima Grade, Fisher) resulting in a $100 \mu \mathrm{M}$ stock solution. The stock solution was kept at $-4{ }^{\circ} \mathrm{C}$ and protected from light. For optical studies, the stock solution was diluted $1 / 10$ in filtered PBS to create a 10 $\mu \mathrm{M}$ Prodan solution.

B. Tryptophan Steady-State and Lifetime Studies. For each antibody studied, a solution of $0.5 \mathrm{mg} / \mathrm{mL}$ antibody $(2.9 \mu \mathrm{M})$ in PBS was filtered using a $0.2 \mu \mathrm{m}$ Acrodisc syringe filter. Protein concentrations were measured in $3 \times 3 \times 43 \mathrm{~mm}$ quartz cuvettes by double-beam UV-vis spectroscopy (Perkin-Elmer Lambda 2) using the absorption at $280 \mathrm{~nm}\left(\epsilon_{280}=1.35\right.$ (mg/

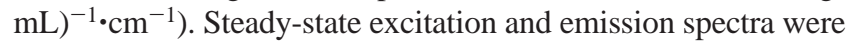
collected using an ISS K2 multifrequency phase fluorometer. Excitation spectra were recorded by monitoring the fluorescence signal at the emission maximum (approximately $330 \mathrm{~nm}$ ) while scanning the excitation wavelength. A xenon arc lamp set at a current of 10 A was used as the excitation source; a monochromator with a dispersion of $8 \mathrm{~nm} / \mathrm{mm}$ was used to select the excitation frequency. The emission spectra were recorded by exciting the solution at the excitation maximum (approximately $285 \mathrm{~nm}$ ) and collecting the fluorescence signal through a monochromator with a dispersion of $8 \mathrm{~nm} / \mathrm{mm}$. The slit widths of both the excitation and emission monochromators were $0.5 \mathrm{~mm}$ in all cases. A dilution correction was applied to the spectra obtained after addition of hapten to an antibody solution.

For lifetime studies, the antibody solution and a glycogen reference solution were placed in the ISS K2 multifrequency phase fluorometer. The xenon arc lamp current was set to 15 A, and the excitation monochromator (slit width $0.5 \mathrm{~mm}$ ) was used to select an excitation wavelength of $285 \mathrm{~nm}$. The total emission was collected without any filter due to a long lifetime component introduced by the filter. Frequency domain data were collected at between 10 and 15 modulation frequencies from 2 to $160 \mathrm{MHz}$. After lifetime measurements were made on the free antibody, hapten solution was added to give a final hapten concentration of $5.9 \mu \mathrm{M}$. Studies were done on fully mature antibodies $38 \mathrm{C} 2$ and $84 \mathrm{G} 3$, tertiary antibody 3.22, secondary antibody 2C26.1, and primary antibody A.3.1.1. The cuvette was agitated by vortexing and allowed to equilibrate for $5 \mathrm{~min}$. Frequency domain data were then collected under the same conditions used before hapten addition at 10-15 modulation frequencies from 2 to $160 \mathrm{MHz}$. After the frequency domain data were collected for a system, analysis was performed using the ISS Lifetime Decay Analysis software package.

This software package allows the lifetime data to be fit to a sum of exponentials or to a sum of lifetime distributions. It is more typical to assume a sum of discrete lifetime values, giving the following expression for the decay of the fluorescence intensity:

$$
I(t)=\sum_{i} \alpha_{i} \exp \left(-t / \tau_{i}\right)
$$

where $\tau_{i}$ is the fluorescence lifetime of the $i$ th component and $\alpha_{i}$ is the preexponential factor for the $i$ th component. The fractional contribution $\left(f_{i}\right)$ of each component to the total fluorescence is given by

$$
f_{i}=\frac{\alpha_{i} \tau_{i}}{\sum_{i} \alpha_{i} \tau_{i}}
$$

If the fluorophore under investigation exists in a variety of different protein environments, it is possible that rather than a sum of discrete fluorescence lifetime values, a continuous distribution of lifetime values may more accurately describe the system. This type of analysis has been applied previously to the study of tryptophan lifetimes in proteins. ${ }^{12,24-26}$ The intensity decay is then given by the following

$$
I(t)=\int_{\tau=0}^{\infty} \alpha(\tau) \exp (-t / \tau) \mathrm{d} \tau
$$

where $\int \alpha(\tau) \mathrm{d} \tau=1$. The fitting scheme that minimized the value of the reduced $\chi^{2}\left(\chi_{\mathrm{R}}^{2}\right)$ goodness-of-fit parameter was taken to be the most realistic decay scheme. In the cases where a distribution provided the minimum value of $\chi_{\mathrm{R}}{ }^{2}$, the functional form of the distribution was a Gaussian, giving the following for $\alpha(\tau)$

$$
\alpha(\tau)=\frac{1}{\sigma(2 \pi)^{1 / 2}} \cdot \exp \left[-\frac{1}{2}\left(\frac{\tau-\tau^{\prime}}{\sigma}\right)^{2}\right]
$$

where $\tau^{\prime}$ is the central value of the distribution and $\sigma$ is the standard deviation of the distribution. For a distribution, the fractional contribution to the overall fluorescence intensity $\left(f_{i}\right)$ is given by

$$
f_{i}=\frac{\int_{0}^{\infty} f_{i}(\tau) \tau \mathrm{d} \tau}{\int_{0}^{\infty} \sum_{i} f_{i}(\tau) \tau \mathrm{d} \tau}
$$

The analysis described above yielded results identical to a similar analysis performed using the Globals Unlimited lifetime decay software. ${ }^{27}$ For each antibody, the overall combination of discrete lifetimes and distributional fits that yielded the lowest value of $\chi_{\mathrm{R}}{ }^{2}$ was recorded. In most trials, a single discrete scatter component was added at $0 \mathrm{~ns}$ to account for the natural scattering of light caused by any solution.

C. Prodan Steady State and Lifetime Studies. Steady state and lifetime measurements were done using an ISS K2. To compare the steady-state spectra of free Prodan and Prodan bound to an antibody, a Prodan concentration of $20 \mathrm{nM}$ was used. All other studies (steady state and lifetime) were done at a Prodan concentration of $1.0 \mu \mathrm{M}$. Concentrations of antibody were used (based on measured binding constants, data not shown) to ensure that $>95 \%$ of the Prodan present was bound. Complete binding occurred within $5 \mathrm{~min}$ as determined by steady state spectral changes. Excitation and emission spectra were acquired as described in section B. Lifetime measurements were taken for free Prodan and Prodan bound to all antibodies studied with a commercially available nonspecific antibody (40 $\mu \mathrm{M}$ in PBS) as a control. The excitation wavelength used depended on the system studied; the wavelength at which the peak in the excitation spectrum occurred was used in all cases. Frequency domain data were collected at 10-15 frequencies from 2 to $180 \mathrm{MHz}$, and the data obtained were analyzed as described in section $\mathrm{B}$.

\section{Results and Discussion}

A. Tryptophan Measurements. Trp free amino acid in solution at $\mathrm{pH} 7.2$ exhibits a broad fluorescence emission with 


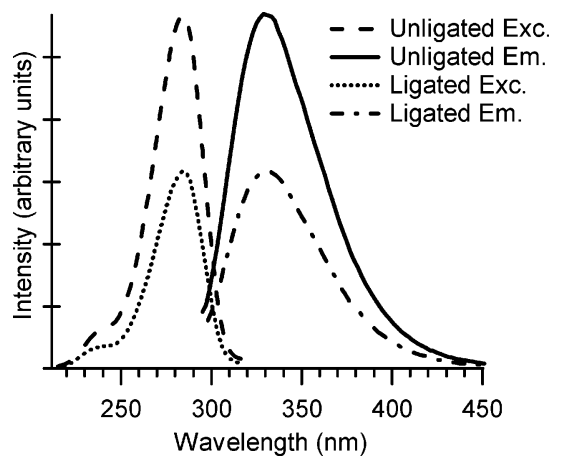

Figure 3. Fluorescence excitation and emission spectra from Trp residues in $2.9 \mu \mathrm{M} 38 \mathrm{C} 2$ mature antibody in the absence (unligated) and presence (ligated) of $5.9 \mu \mathrm{M}$ hapten in PBS, pH 7.2, at $25.0^{\circ} \mathrm{C}$.

a maximum at $352 \mathrm{~nm}$ when excited at $285 \mathrm{~nm}$. The antibodies under investigation possess 24-28 Trp residues, 10-12 of which are at the binding sites. ${ }^{28}$ As shown in Figure 3, the peak of the steady-state emission spectrum from the ensemble of Trp in the mature antibody $38 \mathrm{C} 2$ appears at $330 \mathrm{~nm}$, which is blueshifted relative to Trp in solution. This observation is typical of proteins containing buried tryptophan residues. ${ }^{29}$ The blue shift, similarly, arises from the fact that the energy of the polar excited state $\left({ }^{1} \mathrm{~L}_{\mathrm{a}}\right)$ of $\operatorname{Trp}$ is destabilized in the relatively nonpolar interior of the protein. ${ }^{30}$ Figure 3 also shows hapten-dependent quenching of the Trp fluorescence intensity with no shift in the peak emission wavelength. Control studies in which hapten was added to free Trp in solution and the nonspecific antibody produced negligible quenching of fluorescence (data not shown). It is reasonable to assume that hapten binding to the specific antibodies should affect the fluorescence signal from Trp at the binding site (binding-site Trp) and not Trp far removed from the binding site (framework Trp). This is consistent with the observation that quenching of the Trp signal is not complete. The residual signal intensity is most likely due to these framework Trp residues. The observation that for $38 \mathrm{C} 2$ the fluorescence intensity is quenched nearly $60 \%$ by hapten when only $42 \%(10 / 24)$ of the Trp residues are at the binding site suggests that the binding-site Trp residues have a greater contribution to the overall signal.

Though the general phenomenon of hapten-dependent quenching is similar for the various antibodies studied, details differ. First, the dependence of the quenching on hapten concentration differs, as one would expect, with the quenching being nearly stoichiometric for the tight binders (mature and tertiary) and less complete as we move to secondary and primary antibodies. The low fluorescence quantum yield of Trp $(\approx 0.18)^{31}$ together with the high binding affinities of the antibodies studied preclude an accurate determination of the binding constants from these data. Each antibody also exhibits a slightly different extent of quenching, which again is not surprising. As mentioned above, the antibodies have slight variations in the number of bindingsite and framework Trp residues.

The free amino acid Trp exhibits two discrete lifetimes at 0.68 and $2.77 \mathrm{~ns}$ in PBS at $\mathrm{pH} 7.2$ when excited at $285 \mathrm{~nm}$ (Table 1). The origin of these two lifetimes for Trp in solution is generally believed to be due to the presence of rotamers involving different rotations of the indole ring over the zwitterionic charges of the free amino acid. ${ }^{25,32}$ A review of the fluorescence lifetimes from single Trp containing proteins reveals that some have single lifetimes that vary from 1 to 10 ns, and others have two lifetime components, as observed for Trp in solution. ${ }^{33}$ The origin of the two lifetimes observed in proteins in which peptide bond formation precludes the zwit- terionic form of the free amino acid is still a matter of debate..$^{25,34}$ The best fits to our phase and modulation data (shown in Figure 4 for 38C2, solid lines, and summarized for all antibodies examined in Table 1) reveal two Trp lifetimes: one $\left(\tau_{\mathrm{S}}\right)$ between 0.24 and $1.00 \mathrm{~ns}$, which makes up approximately $20-30 \%$ of the total signal, and one $\left(\tau_{\mathrm{L}}\right)$ between 2.76 and $3.63 \mathrm{~ns}$, which accounts for $60-70 \%$ of the total signal (the remaining $10 \%$ of the intensity is a scattering component at $0 \mathrm{~ns}$ ). The long lifetime component $\left(\tau_{\mathrm{L}}\right)$ of these antibodies in the absence of hapten varies from 3.32 to 3.49 ns at a fractional contribution of $55-70 \%$. The phase and modulation data for the long lifetime component of Trp in the antibodies is best fit to a Gaussian distribution of lifetimes, from which we obtain a lifetime distribution with a full width at half-maximum of $0.42-1.26$ ns. It is interesting to note that in the absence of hapten the width of the lifetime distribution decreases as the maturity of the antibody studied increases.

As hapten is added to all five specific antibodies, fluorescence intensity quenching is observed. With the exception of $38 \mathrm{C} 2$, the value of $\tau_{\mathrm{L}}$ observed for each antibody remains essentially unchanged upon addition of hapten, though the range of $\tau_{\mathrm{L}}$ values observed $(3.13-3.63 \mathrm{~ns})$ is larger. For 38C2, when hapten is added the value of $\tau_{\mathrm{L}}$ decreases significantly, from 3.38 to $2.76 \mathrm{~ns}$. In every case, the fractional intensity of the long lifetime component remains between $55 \%$ and $70 \%$. The full width at half-maximum of the lifetime distributions in the presence of hapten vary from 0.41 to $1.22 \mathrm{~ns}$. If it is true that immature antibodies have a great deal of conformational flexibility, whereas mature antibodies are more conformationally limited, we would expect the distribution widths of the antibodies to decrease as a function of antibody maturity. As seen from the data in Table 1, a general trend of more narrow distributions for the more mature antibodies is indeed observed. Additionally, we would expect the distribution width of mature antibodies to remain relatively constant upon hapten binding if the binding sites for mature antibodies are restricted to conformations that favor specific hapten binding. This behavior is observed for $38 \mathrm{C} 2$ (but not for 84G3), which shows a lack of any appreciable change in the distribution width when hapten is bound. This is in contrast to the primary and secondary antibodies, which exhibit a marked change in distribution width upon hapten binding. Limitations in our equipment preclude an accurate determination of any lifetime below $1 \mathrm{~ns}$, so we are hesitant to interpret the changes observed in the short lifetime $\left(\tau_{\mathrm{S}}\right)$ component. In every case, the best fit gave a discrete value for $\tau_{\mathrm{S}}$.

B. Prodan Binding to Antibodies. Prodan binds to all antibodies elicited against the diketone hapten, including $38 \mathrm{C} 2$, 84G3, and the primary, secondary, and tertiary antibodies cloned and expressed in our lab with binding constants ranging from $5 \mu \mathrm{M}$ (primary) to $125 \mathrm{nM}$ (84G3) (data not shown). This relatively tight binding can be understood by comparison of the molecular structures of the two molecules shown in Figure 2 . Both molecules contain an aromatic system with a derivatized nitrogen seven carbons removed from a ketone.

The excitation and emission spectra of both free Prodan and Prodan bound to 38C2 are shown in Figure 5. The observation of a large shift of the fluorescence emission maximum from 530 (Prodan in solution) to $452 \mathrm{~nm}$ (Prodan bound to 38C2) is characteristic of Prodan's movement from a highly polar solvent to a less polar protein binding site, and has been reported previously. ${ }^{9,15-20}$ Addition of the hapten to a solution of antibody with Prodan bound causes the emission maximum to shift back to $530 \mathrm{~nm}$ and lose intensity (data not shown). This indicates 
TABLE 1: Tryptophan Lifetimes and Lifetime Distributions from Multifrequency Phase Fluorometry ${ }^{a}$

\begin{tabular}{|c|c|c|c|c|c|c|c|c|}
\hline \multicolumn{2}{|c|}{ system } & $\tau_{\mathrm{L}}(\mathrm{ns})$ & $\mathrm{fw}_{\mathrm{L}}^{b}(\mathrm{~ns})$ & $f_{L}$ & $\tau_{\mathrm{S}}(\mathrm{ns})$ & $\mathrm{fw}_{\mathrm{s}}^{b}(\mathrm{~ns})$ & $f_{S}$ & $\chi_{\mathrm{R}}^{2}$ \\
\hline \multirow[t]{2}{*}{$38 \mathrm{C} 2$} & mature & $3.38(0.46)^{c}$ & $0.42(0.06)$ & 0.55 & $1.00(0.53)$ & $\mathrm{d}^{d}$ & 0.26 & 2.0 \\
\hline & +hapten & $2.76(0.25)$ & $0.41(0.04)$ & 0.55 & $0.29(0.17)$ & $\mathrm{d}$ & 0.37 & 2.2 \\
\hline \multirow[t]{2}{*}{$84 \mathrm{G} 3$} & mature & $3.34(0.17)$ & $0.98(0.05)$ & 0.63 & $0.55(0.29)$ & $\mathrm{d}$ & 0.20 & 1.9 \\
\hline & +hapten & $3.43(0.02)$ & $0.67(0.27)$ & 0.54 & $0.71(0.07)$ & d & 0.21 & 1.5 \\
\hline \multirow[t]{2}{*}{3.22} & tertiary & $3.49(0.13)$ & $1.03(0.16)$ & 0.71 & $0.49(0.23)$ & $\mathrm{d}$ & 0.23 & 1.5 \\
\hline & +hapten & $3.13(0.07)$ & $1.22(0.13)$ & 0.71 & $0.24(0.03)$ & d & 0.28 & 1.5 \\
\hline \multirow[t]{2}{*}{$2 \mathrm{C} 26.1$} & secondary & $3.32(0.27)$ & $1.13(0.08)$ & 0.59 & $0.74(0.18)$ & d & 0.27 & 1.5 \\
\hline & +hapten & $3.63(0.11)$ & $0.88(0.39)$ & 0.55 & $0.70(0.26)$ & $\mathrm{d}$ & 0.29 & 2.6 \\
\hline \multirow[t]{4}{*}{ A3.1.1 } & primary & $3.42(0.12)$ & $1.26(0.04)$ & 0.61 & $0.98(0.13)$ & $\mathrm{d}$ & 0.22 & 1.8 \\
\hline & +hapten & $3.18(0.32)$ & $1.18(0.10)$ & 0.62 & $0.47(0.33)$ & d & 0.27 & 1.7 \\
\hline & tryptophan & $2.77(0.09)$ & d & 0.89 & $0.68(0.29)$ & $\mathrm{d}$ & 0.11 & 2.0 \\
\hline & +hapten & $2.67(0.08)$ & d & 0.89 & $0.48(0.34)$ & $\mathrm{d}$ & 0.11 & 1.4 \\
\hline
\end{tabular}

${ }^{a}$ Protein concentrations are $3.3 \mu \mathrm{M}$ for antibody solutions and $2.9 \mu \mathrm{M}$ for antibody/hapten solutions in PBS, pH 7.2 , at $25.0^{\circ} \mathrm{C}$. Trp concentration was $90 \mu \mathrm{M}$, which is approximately the same as the overall Trp concentration of the antibody solutions used for the antibody trials. ${ }^{b}$ The full width at half-maximum values of the Gaussian lifetime distributions, equal to $2.35 \sigma .{ }^{c}$ Standard deviations are reported in parentheses next to the lifetime and distribution width values. ${ }^{d}$ The letter $\mathrm{d}$ is used to indicate that a discrete lifetime provided the best fit to the data. Short lifetime components always fit best to a discrete lifetime.

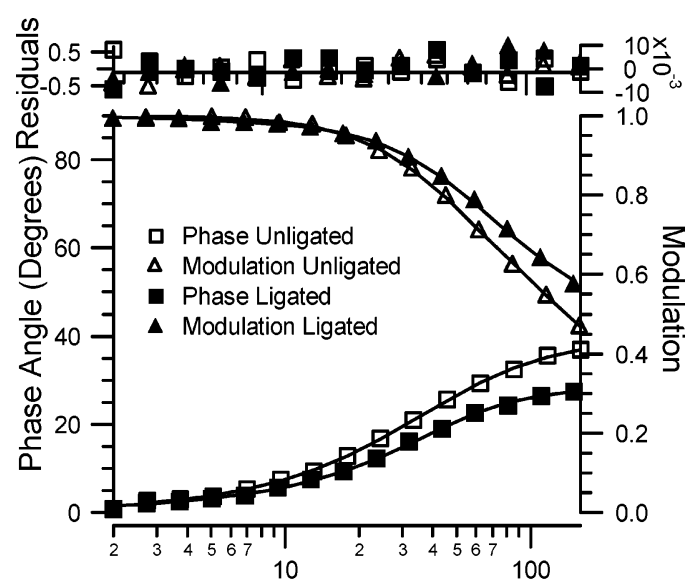

Modulation Frequency $(\mathrm{MHz})$

Figure 4. Multifrequency phase fluorometry of Trp residues of the $38 \mathrm{C} 2$ mature antibody in PBS, $\mathrm{pH} 7.2$, in the absence (unligated) and presence (ligated) of hapten at $25.0^{\circ} \mathrm{C}$.

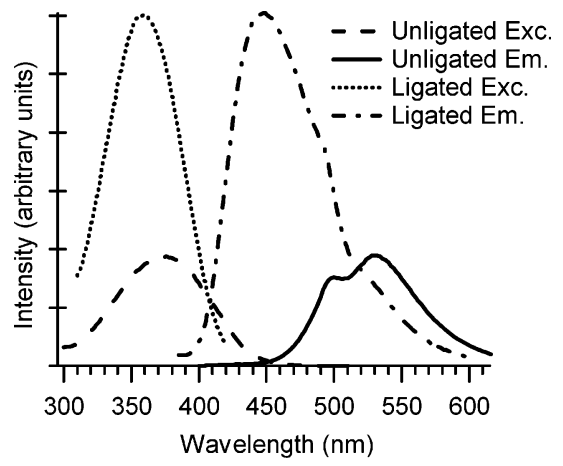

Figure 5. Excitation and emission spectra of $20 \mathrm{nM}$ Prodan in PBS, $\mathrm{pH} 7.2$, at $25.0^{\circ} \mathrm{C}$ in the absence (unligated) and presence (ligated) of the $38 \mathrm{C} 2$ mature antibody.

that Prodan is displaced from the binding site by the hapten, demonstrating that Prodan binds specifically in the antibody binding sites. The excitation and emission spectra were recorded for each antibody as well as a nonspecific antibody, which did not bind to Prodan, and the results obtained are summarized in Table 2. Prodan fluorescence anisotropy titration curves (unpublished data) indicate that the two mature antibodies bind tightly, that binding is weaker for the tertiary and the primary antibodies and, finally, that the secondary antibody binds most weakly. The nonspecific antibody does not bind Prodan, as evidenced by the lack of any change in the emission maximum.
TABLE 2: Prodan Steady-State Fluorescence Results ${ }^{a}$

\begin{tabular}{llccc}
\hline & system & $\begin{array}{c}\text { exc. } \\
\max (\mathrm{nm})\end{array}$ & $\begin{array}{c}\mathrm{em} . \\
\max (\mathrm{nm})\end{array}$ & $\begin{array}{c}\text { Stokes } \\
\text { shift }\left(\mathrm{cm}^{-1}\right)\end{array}$ \\
\hline Prodan & no antibody & 375 & 528 & 7730 \\
+38C2 & mature & 360 & 452 & 5650 \\
+84G3 & mature & 375 & 473 & 5530 \\
+3.22 & tertiary & 379 & 461 & 4700 \\
+2C26.1 & secondary & 380 & 494 & 6070 \\
+A3.1.1 & primary & 389 & 461 & 4020 \\
+IgG & nonspecific & 375 & 531 & 7830
\end{tabular}

${ }^{a}$ Prodan concentration was $1.0 \mu \mathrm{M}$ in PBS, pH 7.2, at $25.0^{\circ} \mathrm{C}$. Antibody was added to $>95 \%$ saturation.

Prodan in aqueous solution shows a large Stokes shift of approximately $7700 \mathrm{~cm}^{-1}$, which has been attributed to fluorescence from a charge-separated excited state that is stabilized by the polar solvent. ${ }^{9}$ The emission of Prodan bound to all of the antibodies is blue shifted relative to Prodan in solution, as shown by a decrease in the Stokes shift to $4000-6000 \mathrm{~cm}^{-1}$. The primary and tertiary antibodies show the smallest Stokes shifts (4000 and $4700 \mathrm{~cm}^{-1}$, respectively) suggesting that these sites are the most hydrophobic.

Lifetimes and lifetime distributions as measured by multifrequency phase fluorometry of Prodan in solution and bound to the five specific and one nonspecific antibody are summarized in Table 3. Figure 6 shows the phase shift and modulation as a function of frequency for both Prodan in solution and Prodan bound to the $38 \mathrm{C} 2$ mature antibody. The best fits to our phase and modulation data produce two lifetime components for Prodan: one $\left(\tau_{\mathrm{S}}\right)$ is $<1 \mathrm{~ns}$ and makes up $20-60 \%$ of the total signal and one $\left(\tau_{\mathrm{L}}\right)$ is between 1.7 and $5.0 \mathrm{~ns}$ and accounts for $40-80 \%$ of the total signal. An accurate determination of the short lifetime component is beyond the resolution of our equipment, and this component could not be resolved from the scattering signal. In every case, the best fit to the data produced a discrete value for $\tau_{\mathrm{S}}$. The long lifetime components $\left(\tau_{\mathrm{L}}\right)$ of Prodan in solution (1.81 ns) and bound nonspecifically to the control antibody (1.66 ns) are very similar. The secondary antibody, which binds Prodan only weakly, shows a slightly longer lifetime $(2.90 \mathrm{~ns})$ and a very large distribution width. The primary, tertiary, and the two mature antibodies show much longer Prodan lifetimes, ranging from 4.03 to $5.03 \mathrm{~ns}$, suggesting that coupling from the locally excited state to the chargeseparated state is less efficient when Prodan is bound to these antibodies. The lifetime distributions of Prodan bound to the antibody are most wide for the weakest-binding secondary antibody and become increasingly narrow for the more mature 
TABLE 3: Prodan Lifetimes and Lifetime Distributions from Multifrequency Phase Fluorometry ${ }^{a}$

\begin{tabular}{|c|c|c|c|c|c|c|c|c|}
\hline \multicolumn{2}{|c|}{ system } & $\tau_{\mathrm{L}}(\mathrm{ns})$ & $\mathrm{fw}_{\mathrm{L}}^{b}(\mathrm{~ns})$ & $f_{\mathrm{L}}$ & $\tau_{\mathrm{S}}(\mathrm{ns})$ & $\mathrm{fw}_{\mathrm{s}}{ }^{b}$ & $f_{\mathrm{S}}$ & $\chi_{\mathrm{R}}^{2}$ \\
\hline Prodan & no antibody & $1.81(0.06)^{c}$ & $\mathrm{~d}^{d}$ & 0.42 & $0.40(0.20)$ & $\mathrm{d}$ & 0.57 & 1.4 \\
\hline$+38 \mathrm{C} 2$ & mature & $4.16(0.03)$ & $0.77(0.06)$ & 0.80 & $0.11(0.07)$ & $\mathrm{d}$ & 0.20 & 1.2 \\
\hline$+84 \mathrm{G} 3$ & mature & $5.03(0.012)$ & $0.96(0.31)$ & 0.78 & $0.20(0.30)$ & $\mathrm{d}$ & 0.20 & 1.6 \\
\hline+3.22 & tertiary & $4.03(0.05)$ & $1.00(0.23)$ & 0.46 & $0.01(0.01)$ & $\mathrm{d}$ & 0.54 & 1.4 \\
\hline$+2 \mathrm{C} 26.1$ & secondary & $2.90(0.02)$ & $1.86(0.03)$ & 0.38 & $0.03(0.02)$ & $\mathrm{d}$ & 0.62 & 1.3 \\
\hline +A3.1.1 & primary & $4.72(0.03)$ & $0.91(0.13)$ & 0.40 & $0.01(0.01)$ & $\mathrm{d}$ & 0.60 & 1.7 \\
\hline$+\operatorname{IgG}$ & nonspecific & $1.66(0.07)$ & $\mathrm{d}$ & 0.40 & $0.12(0.02)$ & d & 0.60 & 1.8 \\
\hline
\end{tabular}

${ }^{a}$ Prodan concentration was $1.0 \mu \mathrm{M}$ in PBS, $\mathrm{pH} 7.2$, at $25.0{ }^{\circ} \mathrm{C}$. Antibody was added to $>95 \%$ saturation. ${ }^{b}$ Full width at half-maximum values of the Gaussian fits to the lifetime distributions, equal to $2.35 \sigma{ }^{c}$ Standard deviations are reported in parentheses next to the lifetime and distribution width values. ${ }^{d}$ The letter $\mathrm{d}$ is used to indicate that a discrete lifetime provided the best fit to the data. Short lifetime components always fit best to a discrete lifetime.

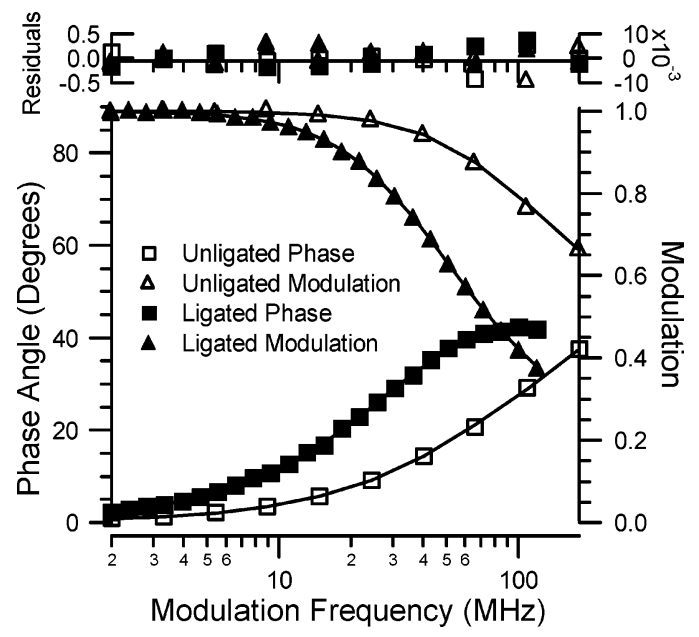

Figure 6. Multifrequency phase fluorometry of $1.0 \mu \mathrm{M}$ Prodan in PBS, $\mathrm{pH} 7.2$, in the absence (unligated) and presence (ligated) of $38 \mathrm{C} 2$ mature antibody at $25.0^{\circ} \mathrm{C}$.

antibodies. These data are consistent with a decrease in the number of conformations accessible to Prodan in the antibody binding site as the antibody maturity increases.

\section{Conclusions}

Because the intrinsic Trp fluorescence lifetimes $\left(\tau_{\mathrm{L}}\right)$ for the five antibodies we studied are remarkably similar to one another, and not sensitive to the affinity maturation state of the antibody, they cannot be used to obtain information about the conformational heterogeneity of the binding site. This may be due to the complexity of the system, and the fact that approximately $58 \%$ of the Trp that make up the signal are not at the binding sites. Experiments in progress to express single chain antibodies, in which only the N-terminal variable region is present, are sure to yield more definitive results. Although the lifetimes themselves do not change as a result of affinity maturation, the width of the lifetime distributions do vary with the maturity of the antibody. The widths of the long lifetime distributions, which were obtained from a Gaussian fit to the phase and modulation data, become increasingly narrow in the antibodies elicited after multiple exposures to antigen. Despite the large quenching of the intensity of the Trp signal upon hapten binding, the changes in the fluorescence lifetimes $\left(\tau_{\mathrm{L}}\right)$ of the antibodies upon binding hapten are quite small, with the exception of $38 \mathrm{C} 2$. Not surprisingly, the distribution width narrows upon hapten binding. This feature is most significant for the primary and secondary antibodies, and smallest for the mature 38C2 antibody. These results are consistent with the hypothesis that the process of affinity maturation produces antibodies with fewer accessible conformational states so as to reduce the entropic cost to the antibody when hapten binds. ${ }^{2-5}$
To complement these observations, the fluorescence from Prodan, a hapten analogue that binds in the antibody binding site, is quite sensitive to the maturation state of the antibody. Emission maxima, lifetimes, and lifetime distributions depend on whether the antibody has been obtained from mice that have had little or multiple exposures to hapten. The increase of the fluorescence lifetime and the decrease of the Stokes shift of Prodan as a function of antibody maturity indicate that the ligand becomes increasingly shielded from solvent. The decrease in the width of both the Trp and Prodan lifetime distributions observed for the series of antibodies studied results from a decrease in the conformational heterogeneity of the system examined. This could be a result of a loss of flexibility in the antibody binding sites, or simply a reduction in the number of conformational states of the binding sites. To distinguish between these two possibilities, the technique of time-resolved fluorescence anisotropy must be used. These experiments are currently underway in our laboratory.

Acknowledgment. This work is inspired by Richard Bersohn, who made ground-breaking contributions to biophysics before the term had gained widespread use. His vision that complicated systems should obey the same laws of physics as smaller molecules and his capacity to make bold suggestions and brilliant connections has inspired our own life-long interest in biophysical chemistry. We would also like to thank David Hansen and Nalini Shah-Mahoney for synthesizing the hapten, Richard Goldsby for the immunological work of creating the library of monoclonal antibodies, and David Ratner for sequencing the related antibodies. Funding for this work was provided by the National Science Foundation through its Collaborative Research at Undergraduate Institutions C-RUI Program, the Camille and Henry Dreyfus Scholar/Fellow Program for Undergraduate Institutions, and the Faculty Research Awards Program at Amherst College.

\section{References and Notes}

(1) Goldsby, R. A.; Osbourne, B. A.; Kuby, J.; Kindt, T. J. Kuby Immunology, 4th ed.; W. H. Freeman: New York, 2000.

(2) Wedemayer, G. J.; Patten, P. A.; Wang, L. H.; Schultz, P. G.; Stevens, R. C. Science 1997, 276, 1665-1669.

(3) Mundorff, E. C.; Hanson, M. A.; Varvak, A.; Ulrich, H.; Schultz, P. G.; Stevens, R. C. Biochemistry 2000, 39, 627-632.

(4) Yin, J.; Mundorff, E. C.; Yang, P. L.; Wendt, K. U.; Hanway, D.; Stevens, R. C.; Schultz, P. G. Biochemistry 2001, 40, 10764-10773.

(5) Yin, J.; Andryski, S. E.; Beuscher, A. E.; IV.; Stevens, R. C.; Schultz, P. G. Proc. Natl. Acad. Sci. U.S.A. 2003, 100, 856-861.

(6) Furukawa, K.; Akasako-Furukawa, A.; Shirai, H.; Nakamura, H.; Azuma, T. Immunity 1999, 11, 329-338.

(7) Manivel, V.; Sahoo, N. C.; Salunke, D. M.; Rao, K. V. S. Immunity 2000, 13, 611-620.

(8) Wagner, J.; Lerner, R. A.; Barbas, C. F., III. Science 1995, 270, $1797-1800$.

(9) Weber, G.; Farris, F. J. Biochemistry 1979, 18, 3075-3078. 
(10) Dal Porto, J. M.; Haberman, A. M.; Shlomchik, M. J.; Kelsoe, G. J. Immunol. 1998, 161, 5373-5381.

(11) Tetin, S. Y.; Hazlett, T. L. Methods 2000, 20, 341-361.

(12) Lakowicz, J. R.; Cherek, H.; Gryczynski, I.; Joshi, N.; Johnson,

M. L. Biophys. Chem. 1987, 28, 35-50.

(13) Alcala, J. R.; Gratton, E.; Prendergast, F. G. Biophys. J. 1987, 51, $597-604$.

(14) Alcala, J. R.; Gratton, E.; Prendergast, F. G. Biophys. J. 1987, 51, 925-936.

(15) Johnsson N.; Marriott G.; K.; W. EMBO J. 1988, 7, 2435-2442.

(16) Mazumdar, M.; Parrack, P. K.; Bhattacharyya, B. Eur. J. Biochem. 1992, 204, 127-132.

(17) Lasagna, M.; Vargas, V. C.; Jameson, D. M.; Brunet, J. E. Biochemistry 1996, 35, 973-979.

(18) Hiratsuka, T. Biochemistry 1998, 37, 7167-7176.

(19) List, B.; Barbas, C. F., III.; Lerner, R. A. Proc. Natl. Acad. Sci. U.S.A. 1998, 95, 15351-15355.

(20) Krishnakumar, S. S.; Panda, D. Biochemistry 2002, 41, 7443-7452.

(21) Rose, N.; DeMacrio, E.; Fahey, J.; Friedman, H.; Penn, G. Manual of Clinical Laboratory Immunology; American Soc. Microbiology Press: Washington, DC, 1997.

(22) Zhong, G.; Lerner, R. A.; Barbas, C. F., III. Angew. Chem., Int. Ed. 1999, 38, 3738-3741.

(23) Sinha, S. C.; Sun, J.; Miller, G.; Barbas, C. F., III; Lerner, R. A Organic Lett. 1999, 1, 1623-1626.

(24) Vos, R.; Engelborghs, Y.; Izard, J.; Baty, D. Biochemistry 1995, $34,1734-1743$
(25) Lakowicz, J. R. Photochem. Photobiol. 2000, 72, 421-437.

(26) Ellis, J.; Bagshaw, C. R.; Shaw, W. V. Biochemistry 1995, 34, 3513-3520.

(27) Beechem, J. M.; Gratton, E.; Mantulin, W. W. Globals Unlimited Software, R.3. University of Illinois, 1990.

(28) Wang, Z.; Raifu, M.; Howard, M.; Smith, L.; Hansen, D.; Goldsby, R.; Ratner, D. J. Immunol. Methods 2000, 233, 167-177.

(29) Weinryb, I.; Steiner, R. F. Excited States of Proteins and Nucleic Acids; Plenum Press: New York, 1971.

(30) Callis, P. R. Methods Enzymol. 1997, 278, 113-150.

(31) Engelborghs, Y.; Fersht, A. Barnase: Fluorescence Analysis of a Three Tryptophan Protein. In Topics in Fluorescence Spectroscopy: Protein Fluorescence; Lakowicz, J. R., Ed.; Kluwer Academic/Plenum Publishers: New York, 1991; Vol. 6; pp 83-101.

(32) Szabo, A. G.; Rayner, D. M. J. Am. Chem. Soc. 1980, 102, 554563.

(33) Hellings, M.; De Maeyer, M.; Verheyden, S.; Hao, Q.; Van Damme, E. J. M.; Peumans, W. J.; Engelborghs, Y. Biophys. J. 2003, 85, 18941902.

(34) Larsen, O. F. A.; van Stokkum, I. H. M.; Pandit, A.; van Grondelle, R.; van Amerongen, H. J. Phys. Chem. B 2003, 107, 3080-3085.

(35) Kratzin, H. D.; Palm, W.; Stangel, M.; Schmidt, W. E.; Friedrich, J.; Hilschmann, N. Biol. Chem. Hoppe-Seyler 1989, 370, 263-272.

(36) Deisenhofer, J. Biochemistry 1981, 20, 2361-2370.

(37) Padlan, E. A. Mol. Immunology 1994, 31, 169-217. 